\title{
Short-Term Femoral Vein Catheterization Rarely Causes Thrombosis or Bacteremia
}

\author{
Ana Isabel Casanegra, Mo ${ }^{1,2}$ \\ Scott Brannan, MD ${ }^{1,2}$ \\ Ramona Dadu, mo ${ }^{1,2}$ \\ Philip Ong, MD ${ }^{1,2}$ \\ Mitchell Berner, MD ${ }^{1,2}$ \\ Kristopher Spinning, mo ${ }^{1,2}$ \\ Terence Hughes, mo 1,2 \\ Greg Marrinan, $\mathrm{MD}^{1,2}$ \\ Kenneth Zinn, MD ${ }^{1,2}$ \\ Constantine Manthous, MD ${ }^{1,2}$
}

\author{
${ }^{1}$ Bridgeport Hospital, Bridgeport, Connecticut. \\ ${ }^{2}$ Yale University School of Medicine, New Heaven, Connecticut.
}

Disclosure: The authors have no financial or intellectual conflicts of interest relevant to this research. The research was not supported by external funding.

BACKGROUND: Experts and national regulatory bodies have deemed femoral vein catheterization (FVC) unsafe, and recommend avoiding it whenever possible.

OBJECTIVE: To assess rates of catheter-related bloodstream infections (CRBI) and deep venous thrombosis (DVT) complicating FVC.

DESIGN: Prospective observational cohort study.

SETTING: Medical intensive care unit (MICU) of a 350-bed community teaching hospital.

PATIENTS: Consecutive admissions to the MICU during 7 months.

METHODS: Demographic, laboratory and Doppler ultrasound studies were collected on patients receiving large vein catheters (VC) in our MICU. Ultrasound examinations were systematically performed on the day of and 5 to 7 days after removal of FVC.

RESULTS: VC were inserted in 238 (35\% of) patients. Of that total, 217 catheters were in large veins ( $49 \% \mathrm{FVC}, 38 \%$ internal jugular and 13\% subclavian) for an average of 2.7 days for femoral, 5.7 days for internal jugular and 3.6 days for subclavian vein catheters. During 1200 catheter-days, no central VC CRBI was identified. Of 107 FVC, initial and follow-up Doppler studies were performed in 50 patients. A total of $97 \%$ of patients received routine thromboprophylaxis and none had a DVT. Of the 57 patients with initial but no Doppler follow-up at 5 to 7 days following removal, no patient developed clinically detected venous thromboembolism (VTE).

CONCLUSION: Short-term FVC was used safely in our MICU in the setting of thromboprophylaxis. In light of its favorable safety profile for initial resuscitation of critically ill patients, it may be premature to strongly discourage FVC. Journal of Hospital Medicine 2011;6:33-36. ๑ 2010 Society of Hospital Medicine.

KEYWORDS: bloodstream infection, catheterization, deep vein thrombosis, femoral vein.

Central venous catheters (CVC) are routinely used to deliver medications and monitor intravascular pressures of critically ill patients. Experts and national regulatory bodies have questioned the safety of femoral vein catheterization (FVC), and currently recommend against venous access at this site whenever possible. ${ }^{1-3}$ However, a large prospective nonrandomized study has suggested that rates of FVC infections are not higher than jugular or subclavian sites. ${ }^{4}$ Some authors have suggested that increased risk of deep vein thrombosis (DVT) also relatively contraindicates the femoral site. ${ }^{5}$ No study has prospectively examined rates of DVT in patients receiving FVC for short durations ( $<72$ hours). In this brief report, we prospectively examined the rates of catheter-related bloodstream infections (CRBI) and DVT in critically ill patients receiving CVC.

\section{Methods}

This prospective observational cohort study was conducted in the medical intensive care unit (MICU) of Bridgeport Hospital, a 350-bed community teaching hospital. The hospital's Institutional Review Board approved the study and waived the informed consent requirement because it has been the practice for the past decade to favor use of the femoral site for initial resuscitations with very low complication rates. All patients admitted to the MICU between September 1, 2008 and March 31, 2009 were eligible. VC were defined as catheters placed in the jugular, subclavian or femoral veins or peripherally inserted and guided to a central intrathoracic vein (PICC). CVC refers to catheters placed directly into central veins. In early 2008, a hospital-wide initiative was introduced to insert all CVC using the Pronovost check-list. ${ }^{1}$ VC sites 
were chosen at the discretion of caregivers in the emergency department and MICU. The policy of our intensive care units is to use only saline flushes of VCs.

Demographic data including age, gender, and body mass index, were collected on all patients. In addition the following parameters were monitored for the duration of ICU stay for the purpose of this study: (1) site and duration of installation of all intravascular catheters, (2) level of training of clinician inserting CVC, (3) catheter/blood culture results. For the purposes of this study, bilateral femoral Doppler compression ultrasound studies were expected to be performed by radiology house officers within 24 hours of removing and again 5 to 7 days following removal of FVC. Local VC complications, methods of thromboprophylaxis and risk factors for venous thromboembolism (VTE) were recorded. Patient outcomes and disposition destinations were also recorded.

CRBI were defined using the Centers for Disease Control definitions. $^{2}$ CRBI were identified by daily review of all positive blood cultures and review of patients' medical records. In addition, Infection Control Committee data were reviewed to corroborate contemporaneously determined CRBI during the study period and for 1 year prior to the study period. Patients with FVC were examined each day for signs or symptoms of thrombosis (tenderness along the vein, leg swelling, pitting edema or visible collateral superficial veins). Patients were followed up until death or hospital discharge for clinical signs, symptoms or diagnosis of thromboembolic disease.

Bedside Duplex ultrasounds of bilateral common femoral and superficial femoral veins were performed using graded compression and color Doppler techniques. The leg without FVC served as the control. Evaluations were conducted by senior radiology residents ( $>100$ hours training) utilizing a high-resolution ( $>7.5 \mathrm{MHz}$ ) linear array transducer. Frame capture images were digitally stored and subsequently reviewed by a Board-certified radiologist, who was blinded to side of insertion and clinical outcomes, and rendered a final interpretation.

Values are listed as means \pm standard deviations. Comparisons of group means were performed using nonpaired Student's $t$ tests. A $P$ value of $<0.05$ signified statistical significance.

\section{Results}

During the study period, 675 patients were admitted to the MICU. VCs were inserted in 238 (35\% of) patients. During their MICU stay, 182 (77\% of) patients had 1 VC, $48(20 \%)$ had $2 \mathrm{VC}$, and 8 (3\%) had 3 VC. On admission, 38 patients (6\%) had preexisting VC (tunneled catheter 58\%, PICC 32\%, and dialysis catheters $10 \%$ ). Additional VCs were placed in 10 of these patients $(26 \%)$.

Of the 302 VC, 85 (28\%) were PICCs and 217 were CVC (107, 49\% FVC; 82, 38\% internal jugular; 28, 13\% subclavian). A total of 151 (28\%) patients had radial arterial cathe- ters placed around the time of admission. The types of CVC included triple lumen in 164 (75\%), dialysis catheters in 29 (13\%), single-lumen large bore catheters in 17 (8\%), and tunneled catheters in 4 patients $(2 \%)$. The average duration ( \pm standard deviation [SD]) of CVC was $2.7 \pm 2.2$ days for FVC, $5.7 \pm 9.6$ days for internal jugular and $3.6 \pm 3.1$ days for subclavian vein catheters.

During these seven months, including 1200 catheterdays, only 1 CRBI was identified in a patient who only had a PICC, yielding an infection rate of 0.83 CRBI per 1000 catheter-days. No femoral, subclavian or internal jugular catheter infections were detected. Hospital epidemiologic data confirmed this finding, and demonstrated only 1 other CRBI during 3721 line-days, in the 7 months of this study and 12 months before, yielding an average of $0.40 \mathrm{CRBI} /$ 1000 catheter-days.

Of 107 FVC, 101 were placed during initial resuscitations and 6 as second-access sites, (2 for dialysis, 4 triple lumen catheters). Thromboprophylaxis was administered to 104 (97\% of) patients with FVC. Thromboprophylaxis was pharmacological (heparins) in 63 (59\% of) patients and mechanical (pneumatic compression) in 46 (43\%). Five patients had both mechanical and pharmacological prophylaxis. Catheters were placed by a critical care or emergency department attending in $11 \%$, critical care fellows in $11 \%$, and residents in $78 \%$. Ultrasound studies of the legs were performed in 57 patients; 56 had studies within 24 hours of removing FVC. Of these 56 patients, 53 studies were interpreted as negative and 3 were considered incomplete. The 3 initially incomplete studies were repeated, and found to be negative. Six patients were discharged from the hospital before the postFVC-removal ultrasound could be performed. Of the 50 patients who had both ultrasounds (initial and follow up 5-7 days after removal of FVC), none had a DVT on the side of the catheter or in the control leg. Of the 50 patients with no ultrasound follow-up, no patient developed clinically detected VTE; these patients had FVC for shorter duration $(2.4 \pm 2.4$ vs. $3.4 \pm 1.9$ days for those with 2 Duplex; $P=0.02$ ) and their ICU length of stay was shorter $(3.8 \pm 4.6$ vs. $6.6 \pm 5.6$ days for those with 2 Duplex; $P=0.01$ ).

Since no VTE or CRBI were detected further analyses regarding risks for these complications was not possible.

\section{Discussion}

Contrary to regulatory guidelines suggesting a poor safety profile, we found that short-term FVC was associated with no episode of DVT or CRBI. While the incidence of complications is lower in more experienced operators, ${ }^{6}$ most FVC in our hospital were placed by resident-trainees $(78 \%)$ with or without supervision from an attending physician. There were no immediate or subacute (ie, thrombosis, infection) major complications. There are a number of features that favor short-term FVC for initial resuscitation of critically ill patients. Subclavian and intrajugular CVC require prolonged Trendelenburg position, which may not be well tolerated by 
some patients. FVC does not require Trendelenburg position. Major bleeding- $1.0 \%$ to $1.5 \%$ for all the CVC-is minimized because direct compression of femoral vessels is possible. Compression of subclavian hemorrhage is impossible while compression of the jugular vessels is uncomfortable. Pneumothorax, while uncommon in the subclavian and intrajugular approaches, ${ }^{7}$ has serious consequences for an unstable patient, whereas FVC obviates the risk. Some might argue that FVC cannot accurately reflect cardiovascular filling thereby defeating 1 of the important purposes of the catheter. While this is certainly true in patients with raised intraabdominal pressures, a small case series suggests that (longer-than-normal) FVC can accurately measure central filling pressures. ${ }^{8}$ Another potential shortcoming of FVC is that if used only for short durations during initial resuscitations - as in this study - some patients will require a second CVC or PICC with incumbent risks.

Our study differs from previous studies that have shown infection rates ranging from $1.5 / 1000$ to $20 / 1000$ catheterdays $^{4,9,10}$ and thrombosis rates of $6.6 \%$ to $25 \% .^{5,10-13}$ Some previous studies have suggested higher rates of infection of FVC relative to internal jugular or subclavian sites (3.7/1000 vs. $20 / 1000$ catheter-days) ${ }^{9}$ while others found similar infection or colonization rates between femoral and nonfemoral sites. ${ }^{4,10}$ Our 0.83 CRBSI per 1000 catheter-day rate is similar to that of Pronovost et al. ${ }^{1}$ who avoided FVC, whereas it was the preferred site (nearly half of all CVC) in our MICU. The incidence of VTE in critically ill patients ranges from $9 \%$ to $33 \%,{ }^{14,15}$ and CVC are a well recognized risk factor of VTE. ${ }^{5}$ The reported incidence of DVT in patients with CVC varies widely from $3 \%$ to $10 \%$ in subclavian catheters ${ }^{9}$ to $6.6 \%$ to $25 \%$ in FVC. ${ }^{11,12}$ We attribute the remarkable difference in our results to the fact that FVC was used for brief durations (mean 2.7 days, range 1-16 days) for the primary purpose of resuscitating critically ill patients. Also, techniques introduced by Pronovost et al. ${ }^{1}$ to reduce CRBI had permeated our institutional practices by the time of this study; our results match his, of very low rates of CRBI when checklists are employed. In previous studies, FVC was used for extended durations similar to other CVC sites (ranging from 4 to 9.6 days). ${ }^{5,9,12,13,16}$ Additionally, almost all of our patients received VTE prophylaxis whereas rates were variable in previous studies.

This study has several limitations. First, catheter insertion sites were not randomly assigned. This can introduce selection bias. For example, often femoral access is used in more unstable patients ${ }^{4}$ who are less tolerant of Trendelenberg position whereas it is often avoided in obese patients. Another important limitation is that ultrasound studies were not performed in $47 \%$ of patients who had FVC. While these missed cases were not advertent (eg, CVC on weekends when no study personnel available), we cannot exclude the possibility of bias. However, no FVC patients who did not have ultrasounds developed clinically detected VTE. It is also possible that DVT could have appeared $>5$ to 7 days after our follow-up ultrasound, though later development might favor spontaneous DVT unrelated to CVC. Finally, this was a relatively small study, but it appears that the rate of DVT from FVC, if placed for short durations and accompanied by thromboprophylaxis, is very low.

In conclusion, short-term FVC was used safely-with no major complications-in our MICU. Our data support that short-term FVC (with thromboprophylaxis) has a reasonable safety profile for initial resuscitation of critically ill patients. Notwithstanding the limitations of our study, we suggest that it may be premature to abandon entirely ${ }^{3,17}$ the use of FVC for resuscitation of critically ill patients. We propose that our data suggest the need for a larger study to examine more definitively the safety profile of short-term FVC.

\section{Address for correspondence and reprint requests:}

Constantine Manthous, Bridgeport Hospital and Yale University

School of Medicine, 267 Grant Street, Bridgeport, CT 06610;

Telephone: 203-384-4581; Fax: 203-384-4294; E-mail:

Pcmant@bpthosp.org; Received 1 September 2009; revision

received 2 February 2010; accepted 17 February 2010.

\section{References}

1. Pronovost P, Needham D, Berenholtz S, et al. An intervention to decrease catheter related bloodstream infections in the ICU. $N$ Engl J Med. 2006; 355:2725-2732.

2. O'Grady NP, Alexander M, Dellinger EP, et al. Guidelines for the prevention of intravascular catheter-related infection. MMWR Website. Available at: http://www.cdc.gov/mmwr/preview/mmwrhtml/rr5110al.htm. Accessed February 2010.

3. Joint Commission. National Accreditation: Hospital Patient Safety Goals. Available at: http://www.jointcommission.org/NR/rdonlyres/31666E86E7F4-423E-9BE8-F05BD1CB0AA8/0/HAP_NPSG.pdf. Accessed February 2010.

4. Deshpande KS, Hatem C, Ulrich HL, et al. The incidence of infectious complications of central venous catheters at the subclavian, internal jugular and femoral sites in an intensive care unit population. Crit Care Med. 2005;33:13-20.

5. Trottier SJ, Veremakis C, O'Brien J, Auer AI. Femoral deep vein thrombosis associated with central venous catheterization: Results from a prospective, randomized trial. Crit Care Med. 1995;23:52-59.

6. Sznajder JI, Zveibil FR, Bitterman H, Weiner P, Bursztein S. Central vein catheterization. Failure and complicagtion rates by three percutaneous approaches. Arch Intern Med. 1986;146:259-261.

7. Ruesch S, Walder B, Tramer MR. Complications of central venous catheters: internal jugular versus subclavian access-a systematic review. Crit Care Med. 2002;30:454-460.

8. Joynt GM, Gomersall CD, Buckley TA, Oh TE, Young RJ, Freebairn RC. Comparison of intrathoracic and intra-abdominal measurements of central venous pressure. Lancet. 1996;347:1155-1157.

9. Merrer J, De Jonghe B, Lefrant JY, et al. Complications of femoral and subclavian venous catheterization in critically ill patients. A randomized controlled trial. JAMA. 2001;286:700-707.

10. Parienti JJ, Thirion M, Megarbane B, et al. Femoral vs jugular venous catheterization and risk of nosocomial events in adults requiring acute renal replacement therapy. A randomized trial. JAMA. 2008;299: 2413-2422.

11. Durbec O, Viviand X, Potie F, Vialet R, Albanese J, Martin C. A prospective evaluation of the use of femoral venous catheters in critically ill adults. Crit Care Med. 1997;25:1986-1989.

12. Durbec O, Viviand X, Potie F, Vialet R, Martin C. Lower extremity deep vein thrombosis: a prospective, randomized, controlled trial in comatose or sedated patients undergoing femoral vein catheterization. Crit Care Med. 1997;25:1982-1985. 
13. Joynt GM, Kew J, Comersall CD, Leung VY, Liu EK. Deep venous thrombosis caused by femoral venous catheters in critically ill adult patients. Chest. 2000;117:178-183.

14. Marik PE, Andrews L, Maini B. The incidence of deep venous thrombosis in ICU patients. Chest. 1997;111:661-664.

15. Cook D, Crowther M, Meade M, et al. Deep venous thrombosis in medical-surgical critically il patients: prevalence, incidence and risk factors. Crit Care Med. 2005;33:1565-1571.
16. Timsit JF, Farkas JC, Boyer JM, et al. Central vein catheter related thrombosis in intensive care patients: incidence, risk factors and relationship with catheter related sepsis. Chest. 1998;114:207-213.

17. Institute for Healthcare Improvement. Optimal catheter site selection, with avoidance of the femoral vein for central venous access in adults. Available at: http://www.ihi.org/IHI/Topics/CriticalCare/IntensiveCare/Changes/ IndividualChanges/OptimalCatheterSiteSelectionwithAvoidanceofFemoral VeinforCentralVenousAccessinAdultPatients.htm. Accessed February 2010. 